\title{
A Study on the Problems faced by Mentally ill Patients living in Mangalore
}

\author{
Meena Monteiro' ${ }^{1}, \&$ Laveena D’Mello ${ }^{2}$ \\ ${ }^{1}$ Associate Professor, MSW Department, School of Social Work, Roshni Nilaya, Mangalore, \\ Karnataka, India \\ ${ }^{2}$ Assistant Professor, Social Work Department, Srinivas Institute of Management Studies, Mangalore, \\ Karnataka, India. \\ E-mail: monteiromeena@yahoo.com
}

Type of the Paper: Medical Case Study.

Type of Review: Peer Reviewed.

Indexed In: OpenAIRE

DOI: http://dx.doi.org/10.5281/zenodo.1004666.

Google Scholar Citation: IJHSP

\section{How to Cite this Paper:}

Monteiro, Meena., \& D’Mello, Laveena. (2017). A Study on the Problems faced by Mentally ill Patients living in Mangalore. International Journal of Health Sciences and Pharmacy (IJHSP), 1(2), 24-30.

DOI: http://dx.doi.org/10.5281/zenodo.1004666.

International Journal of Health Sciences and Pharmacy (IJHSP)

A Refereed International Journal

(C) With Authors.

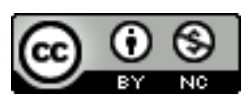

This work is licensed under a Creative Commons Attribution-Non Commercial 4.0 International License subject to proper citation to the publication source of the work.

Disclaimer: The scholarly papers as reviewed and published by the Srinivas Publications (S.P.), India are the views and opinions of their respective authors and are not the views or opinions of the SP. The SP disclaims of any harm or loss caused due to the published content to any party. 


\title{
A Study on the Problems faced by Mentally ill Patients living in Mangalore
}

\author{
Meena Monteiro ${ }^{1}$, \& Laveena D’Mello ${ }^{2}$ \\ ${ }^{1}$ Associate Professor, MSW Department, School of Social Work, Roshni Nilaya, Mangalore, \\ Karnataka, India \\ ${ }^{2}$ Assistant Professor, Social Work Department, Srinivas Institute of Management Studies, Mangalore, \\ Karnataka, India. \\ E-mail: monteiromeena@yahoo.com
}

\begin{abstract}
Psychiatric patients have got an increased morbidity and mortality to other physical health problem. The most problem they are facing is the lifestyle factors such as the use of substances and the decreased physical activity. These patients are mainly restricted to their home. If mental ill patients do not create any problems to the family members and their neighbouring families, the family members are happy and not so much bother about the patients. And if they are not creating any problems for others, family members are not bothered or motivated to treat the mentally ill patients. Hence the lack of motivation from the other family members results in mentally ill patients showing the symptoms of the high risk of being affected by other health problem. There is the availability of treatment to the mentally ill patient. There was only a little availability of the treatment facilities for the psychiatric patients. So the lack of services available and the most vulnerable nature made them more adherent to the problems. There is a direct relationship between the physical and mental health of the patients. In this study the researcher dealing with only the minor issues such as substance abuse, obesity, hypertension, diabetes along with the mental illness, and it would be a way to understand the problems of these patients. The main aim of the study is to study the problems of mentally ill patients and the objectives are; to study the physical health status of the mentally ill patients; and to study the lifestyle factors such as smoking and alcoholism affected the life of mentally ill. The detail studies of 50 intake patients from the hospital where the psychiatric department exist are taken. The interview schedule is used and more observation and case records were referred to get the history. Secondary data is also considered for the research study.
\end{abstract}

Keywords: Mental health, Patients, Diet, Lifestyle, Disease, Substance abuse and diet.

\section{INTRODUCTION :}

Health is considered as the wealth of the nation. All the problems of the nation can be resolved if the health of the individual is increased. According to WHO (World Health Organization) definition health is not only the absence of disease or infirmity; it is the complete physical, mental and social wellbeing. If we consider the mentally ill patients, there are so many problems with the physical health of the mentally ill patients. In order to find out the problems of the mentally ill patients, it is necessary to understand the existing problems of the patients. The main problems of these patients are grouped into problems related to sedimentary lifestyles and the use of substances. In order to find out these problems, we have to assess the health problems of the patients. The present study has gone with the basic problems of obesity, substance abuse and the lifestyle diseases such as hypertension, diabetes etc. [1.2]. These have got a high chance of prevalence among these patients because of their lifestyle factors. So an understanding of these problems can able to explore them more to the life of these patients. Also, the study can able to build among the sample population that they have to be 
checked for these factors also. The problem that exists in the society is that the mentally ill patients can only treat the mental illness; no other treatments were needed for them. This attitude of the family and society must be changed and they must be considered for other problems also [3].

\section{MENTAL ILLNESS AND PHYSICAL HEALTH PROBLEMS :}

The main factors contributing these health problems are the obesity, problems of substance abuse and the lifestyle diseases of diabetes, hypertension, and hyperlipidemia. The present study can assess the problems of the obesity exists in the patients of mentally ill can be noted. Also, the study implies the problems of substance abuse among the mentally ill. The study understands the usage of tobacco or any other forms of substances during the lifespan of the patient. The information of these kinds is mainly obtained from the immediate caretakers of the patient. There has been a various research which compares the results of the finding with the general population and it has been found that the prevalence of these conditions is higher in this group. The problem of physical health was come out from the initial stage. If it will be at the initial stage there won't be any observable cases or the cases that were getting the treatment. In such cases, it will be on the lower side of the iceberg [4].

Mentally ill patients were not getting adequate treatment for the health problems other than mental illness. The problem mainly facing by these patients is that when they are expressing the symptoms it will be taught that they are expressing this due to the mental illness factor. So the actual problem always comes back to the back of the screen. So the problems are not at all going to be identified and thus these problems are not getting the adequate treatment. The researcher only finds out the illnesses which were identified and started the treatment. Also, the researcher was studying to understand the relationship between substance abuse among these individual it is being a fact that substance abuse has a direct retain with the physical health problems [5]. Also, the sedentary lifestyle makes these patients more obese. The mentally ill patients belong to a sedentary lifestyle so that they are being obese in nature and they have got all the lifestyle disorders at a stretch. So the study will identify the problems that were prevalent among these groups which were come out of the mask. The present study goes only on the surface of the main problems of the community.

\section{GENERAL INFORMATION ABOUT MENTAL ILLNESS :}

Mental illness can be defined as a health condition which changes person's thinking or feeling or behavior or all the three and as result of it the person has a distress and difficulty in functioning. The person who was suffering from mental illness may not look sick, especially in minor cases. The mental can be of different types according to the symptoms expressed by the person. The main mental illnesses are schizophrenia, bipolar affective disorder, depression, obsessivecompulsive disorder etc. Mental illnesses are problems with the thinking, behavior and or mood and ability to relate to others. The major mental illness is the depression, schizophrenia, bipolar disorder, panic disorder, obsessivecompulsive disorder, post-traumatic stress disorder and borderline personality disorder. Mental illness can affect any person irrespective of their age, sex, race, economic status etc. The most important thing about mental illness is that the illness which can be treatable [6, 7]. If the person is taking medication on a daily basis the person can able to control the symptoms and can able to live a normal life. In addition to the pharmacological management of the illness therapies especially cognitive behavior therapy, interpersonal therapy etc and peer support group and community support measures can also positively affect the person's illness.

Mental illness was not to be considered as a problem which affects the brain pathology but also it can affect the whole system in which the person lives. It includes the biological, psychological and social parameters. While considering the treatment of the person with mental illness it should not be a whole program which has been based only on the biological nature but should have considered the other factors also. The disease can affect the person in these parameters so there must be treatment options for the person for these structures. The narrow understanding of the 
psychiatric problems with the medical model can only able to find a solution to the brain pathological changes of the person. The other condition must be considered while treating the person as a whole to improve the quality of life of these persons. The challenges in the treatment of the mental illness can also include it should not be focused on the funds derived from the pharmaceutical companies. We all aware of the fact that why these companies were utilizing their funds in this field. Also, we know that now a day the major problem which has been faced by any treatment plan, especially in the psychiatric field, is over medication. Also, these companies were promoting their medicines at any cost. So there should not be any funds utilized from the pharmaceutical companies and if we use these funds it will definitely adverse the action as a whole $[8,9]$.

Mental illness as a whole was considered as a fundamental thing for the person to be considered to the empowering status in the world. On the other hand, women also had the same level. So the results of the both that is the mental illness and the female gender can as a whole affect the person to go to the level which was to be considered to be the deepest of the situation. Females have got the highest risk for developing depression than men. So these kinds of situation in this particular gender type result in various troubles. So it must be considered while treating the illness. The females were more concerned about the family and they were not able to provide the optimum level of care if they were in this type of situation. So the family has to be more problematic than a male person who suffers from the illness. Also, there exists a situation that the married women who found to have been with mental illness they were sent back into their parental home. A person with mental illness was considered to be the most weeks of the persons so they were considered to be the most vulnerable part of the society. Hence the empowerment of this person was in a must. The part of the treatment options available for these groups should not be in any form of discrimination from any point [10]. There will be disabilities associated with the mental illness so they were not having been so many products as it in the last time. It cannot be an easy one to identify the extent of the disability faced by the illness. Also, the illness can have adverse effects on both the person and the family so as a whole these disturbances were also to be considered. If a person with mental illness attempts suicide he would not be considered as a convict for the same.

Mental health problems are a major problem around the world, especially in the developing countries. It affected by the person's life status and how the people were adjusted with their life. The major risk for the mental illness is that if a person in a family was an alcoholic and or using any substance it can definitely affect the other person in the family especially women and children in the mental health set up. In this way, we can definitely state that the mental health can spread from one person to another. Also, the genetic factor plays an important role in the mental illness but also the other factors such as treatment availability, economic status etc. All these will contribute negatively to the person who lives in the developing countries of the world. So these changes were used as a vulnerable among the vulnerable group. The treatment options were not available and even the treatment was available then also they were not able to follow it regularly and hence they were not able to get the desired effect. Mental illness can be treated by collaborative efforts from both the side of the medical team and the family. In the effectiveness of the treatment was determined by how the family influenced by the practice of the effort made by the medical team. The main advantage of the involvement of family in the treatment is that the patient will be available with the family all the time, but the medical team will get that only when they came to the health centre. So the treatment was completely relaid on the family's hands. So the treatment will be effective only when the family has got an understanding of the situation and what were the warning signs of the illness and how the medicines can be utilized etc must be thorough with the family [11, 12]. Family members can be able to explain these things to the medical team. The person should get the benefit of the treatment when the family members are interested in the treatment. The fact is that the person alone can do anything for his benefit. The person should be in complete support from the family members and he cannot do 
anything as alone. So the awareness of the family can improve the quality of care and the treatment of the patient [13].

\section{RELATION WITH OTHER PHYSICAL HEALTH PROBLEMS :}

Patients who were suffering from schizophrenia have got a greater chance of getting the illness such as diabetes hypertension and hypercholesteremia. The treatment of the person with mental illness is not depending on the other characteristics of the patient especially obesity and all because it has got a high chance of relation with the pharmacological medication used for the treatment. The risk of diabetes in people with schizophrenia does not simply stick with the treatment of the antipsychotics but also the lifestyle habits such as lack of exercise, diet habits, and genetic predisposition. A person with a diagnosis of schizophrenia has got a 10 years less than the normal population and in addition to that they were born to have high risk of the problems such as diabetes and obesity because the use of antipsychotics increases weight gain and as result of the weight gain they were more likely don't do exercise. The much-awaited problem of the person who was suffering from mental illness is the fact that these people were not in a situation to understand their condition and seek health advises. Even when they were not a good medical advice they were not able to follow clearly and the care have to be more effective in utilizing in such condition. Otherwise, the problem will not be resolved alone.

Physical activity of the person with mental illness has got a nice relation with the physical health problems of these patients. As a result of the illness, the patient became inactive and the lack of exercise in these patients can definitely add to physical health problems in later life. So the person with mental illness has to be scheduled with the activity which can they followed regularly. In this kind of setup can be administered only with the help of a professional testing with them. As a result of this kind of an activity schedule, they can plan their activity and have a greater chance of getting rid of the physical health problem that was found to be a must at this time. The problem faced by the person with mental illness is that during the treatment they were not getting enough attention to the physical activity part. As a result of these problems, they were in focus with the pharmacological management only. And the caregivers were also happy if they were not making any trouble to others. Care giver's not in a position to understand the importance of the physical activity [13]. These also make it in backward in the action of the physical health management of these patients and they were suffering from a lot of problems especially physical health thereafter.

\section{RELATIONSHIP BETWEEN MENTAL ILLNESS AND SUBSTANCE ABUSE :}

Drug abuse among the people with mental illness is a common problem. It is as higher than about two-fold higher than the normal population. The main reasons for the death of these people were cancer, heart disease, and lung diseases. These are the main causes of the use of tobacco-related substances. There are different reasons for the use of tobacco substances by the people with mental illness. One of the reasons for the user may be to mask the symptoms of the mental illness or in some cases; it may act as a drug to improve the attention and concentration. So they were most addicted to the use and later if they were started to stop the use they were started to have withdrawal symptoms [14].

\section{ANALYSIS :}

Personal Data: The age of the respondents, $58 \%$ were between 21 and 40 years of age, $34 \%$ were between 41 and 60 years of age and $8 \%$ were above 60 years of age. The gender distribution was $50 \%$ were male and $50 \%$ were female. The religious distribution in the sample is that $62 \%$ were Hindus, because more population are Hindus while comparing to other religion. The education status of the sample was $48 \%$ had got the primary education and $36 \%$ had high school education and $16 \%$ are Graduates. The occupation status of the sample population was $42 \%$ were unemployed and $36 \%$ were homemakers and remaining $22 \%$ were working for others or self-employed. The income of $34 \%$ of the sample was below Rs.5,000/-. Remaining had another source of family income. While the researchers assessed the family type in which the patients were living it has been found that 
$49 \%$ of the families were nuclear and $33 \%$ were joint family

Details of Psychiatric illness: While considering the diagnosis of the patients in the sample it has been seen that $18 \%$ were schizophrenia, 64\% have an affective disorder, $14 \%$ were mental retardation with psychotic symptoms and 4\% were with neurotic stressrelated and somatoform disorder. Duration of psychiatric illness of the sample was in such a way that $66 \%$ were above 10 years of duration, $20 \%$ were between 5 and 10, 10\% were between 1 to 4 years and only 4\% have the duration of illness below 1 year. Also, the medications they were taking have been distributed in a way that $76 \%$ of the samples were taking a combination of medications and $22 \%$ were taking only antipsychotics. And 2\% in the sample was only taking an antidepressant.

Physical Health Problems and treatments: The physical health problems were identified for $42 \%$, in which diabetes, hypertension, hepatitis, ulcer and hearing problems. Others had the problem such as kidney problem, thyroid problem, cancer, respiratory problems, epilepsy, pneumonia, and teeth problems. 96\% were taking allopathic treatment for the physical health problems while $2 \%$ are taking Ayurvedic treatment and traditional treatment respectively. About identified cases, only 32\% were on regular treatment and on the prescribed diet while rest $68 \%$ were not at all on regular diet and treatment. Investigator enquired about the details of the investigations they have done. Especially about the investigation for hemoglobin, blood sugar, blood cholesterol and serum electrolytes. It has been found that about $54 \%$ of the sample had their blood checked for haemoglobin and 66\% said that their blood had investigated for blood sugar. 56\% said that their blood had investigated for blood cholesterol and 48\% said that their blood has been investigated for serum electrolytes.

Diet Habits: $20 \%$ of the persons in the sample population prefer vegetarian food, $28 \%$ prefers non-vegetarian food and 52\% prefers mixed diet.

Substance Use, Alcoholic Beverages: The persons were asked regarding the use nicotinecontaining substance in their daily living $34 \%$ were used it and among this $24 \%$ were still using it. And $42 \%$ have not used this kind of substance in their life. In the present study, $66 \%$ had a history of psychiatric illness for more than 10 years 24 were associated with the use of the nicotine-containing substance in their life. 44\% had mental illness between 5 to 10 years.

Physical Activities: When asked regarding some vigorous physical activities that make them sweat $78 \%$ of the sample reported that they were not doing any such activities while $14 \%$ responded that they were doing these type of activity in 1 to 2 times a week and $8 \%$ responded that they were doing it more than 3 times per week. In the other way, the researcher asked about some moderate physical activities than 66\% were doing such activities greater than 5 times a week, $6 \% 3$ to 4 times a week and $14 \% 1$ to 2 times a week. While $14 \%$ were not doing such activities. The person with mental illness was not able to do the vigorous physical activity and the reason for that they had weakness due to the medication. They won't be able to be as fresh as they can when they are with the medication. They can always do some moderate physical activity whenever it was possible. And the duration of the psychiatric illness 66\% were greater than 10 years. That is they were getting the treatment for a long time. They were on the medication for a long time so they are always in the risk of being in the side effects of the medication and that perhaps resulted in the decrease in the physical activity of the persons.

\section{RECOMMENDATIONS SUGGESTIONS :}

AND

The people with mental illness suffer from a lot of problems and they were not getting enough importance. The treatment regularity and the following the prescribed diet was only with $30 \%$ of the population. That is about 70 $\%$ of the population were not in a regular form of treatment for their physical health conditions. The major problem with the treatment of the person with mental illness was that the lack of awareness from the caregivers. They were not interested in the treatment of the other problems of these patients. So there must be provided to identify these problems and should be on regular treatment. Lack of physical activity was another problem identified. It was the use of substances that 
would be harming to the person. There must be appropriate approaches used to give adequate physical activity schedule for the persons and the substance abuse must be taken in to consider and should abolished by the technique used for it.

\section{REFERENCES :}

[1] Acil A. A., Dogan S and Dogan O. (2008). The Effects of Physical Exercises to Mental State and Quality of Life in Patients with Schizophrenia. Journal of Psychiatric and Mental Health Nursing, 15: 808-815.

[2] McCloughen, A. and Foster K. (2011). Weight Gain Associated with Taking Psychotropic Medication: An Integrative Review. International Journal of Mental Health Nursing, 20: 202-222.

[3] Barre L, K. Ferron J. C., Davis K E \& Whitley R. (2011). Healthy Eating in Persons with Serious Mental Illnesses: Understanding and Barriers. Psychiatric Rehabilitation Journal, 34: 304-310.

[4] Chadwick Angelina, Clare Street, Sue McAndrew and Maureen Deacon (2012). Minding our own Bodies: Reviewing the Literature Regarding the Perceptions of Service Users Diagnosed with Serious Mental Illness on Barriers to accessing Physical Health Care. International Journal of Mental Health Nursing, 21: 211-219.

[5] Gazizova, D., Puri, B.K., Singh, L. \& Dhaliwal, R. (2012). The Overweight: Obesity and Plasma lipids in Adults with Intellectual Disability and Mental Illness. Journal of Intellectual Disability Research, 56(9): 895-901.

[6] Gray R. (2012). Physical Health and Mental Illness: A silent Scandal. International Journal of Mental Health Nursing, 21(3): 191-192.

[7] Happell Brenda, Chris Platania-Phung and David Scott (2011). Placing Physical Activity in Mental Health Care: A Leadership Role for Mental Health Nurses. International Journal of Mental Health Nursing, 20: 310-318.
[8] Kessler R.C. (2000). Psychiatric Epidemiology: Selected Recent Advances and Future Directions. Bulletin of the World Health Organization, 78: 464-474.

[9] Lester H.E., Tritter J.Q. \& Sorohan H. (2005). Patients and Health Professionals, Views on Primary Care for People with Serious Mental Illness: Focus Group Study'. British Medical Journal, 330: 1122-1127.

[10] Jorm A.F. (2012). Empowering the Community to Take Action for Better Mental Health. Journal of American Psychologist, 67 (3): 231-243.

[11] Ziller Erika C, Nathaniel J. Anderson \& Andrew F. Coburn (2010). Access to Rural Mental Health Services: Service Use and Out-of-Pocket Costs. The Journal of Rural Health, 26: 214-224.

[12] Laveena D’Mello, Meena monteiro, Sathyendra Prakash (2017) Impact of HIV/AIDS on the Psychosocial Development of Children. International Journal of Health Sciences and Pharmacy (IJHSP), 1(2). 5-10.

[13] Park Tanya, Kim Usher and Kim Foster (2011). Description of a Healthy Lifestyle Intervention for People with Serious Mental Illness Taking Second-generation Antipsychotics. International Journal of Mental Health Nursing, 20: 428-437.

[14] Harris Lynne M, Lynda R Matthews, Jonine Penrose-Wall, Ashraful Alam and Alison Jaworski (2014). Perspectives on Barriers to Employment for Job Seekers with Mental Illness and Additional Substance-use Problems. Health and Social Care in the Community, 22(1): 67-77. 